\title{
What oncology nurses need to know: A study of blood markers, clinic pathologic features, and ovarian cancer prognosis
}

\author{
Xiaoqin $\mathrm{Liu}^{1 \mathrm{1}}$, Yunzhao $\mathrm{Xu}^{1 \Delta}$, Yuquan Zhang ${ }^{1}$, Xiuqun $\mathrm{Xu}^{2^{2 *}}$, Xujuan $\mathrm{Xu}^{{ }^{2 *}}$ \\ ${ }^{1}$ Department of Obstetrics and Gynecology, Nantong University Affiliated Hospital, Nantong, Jiangsu, 226001, China; \\ ${ }^{2}$ Department of Nursing, Nantong University Affiliated Hospital, Nantong, Jiangsu, 226001, China
}

\begin{abstract}
A better understanding of the factors related to cancer helps health professionals like nurses to provide more individualized health care which will affect the prognosis of patients with ovarian cancer. The aim of this study was to analyze the association of clinicopathological features and risk factors with ovarian cancer prognosis. This retrospective study recruited 103 patients with ovarian cancer who were treated at a single institution during the period from May 2002 to May 2014. The blood markers CA125, CA153, and serum ferritin (SF) were detected in all patients before surgery. Risk factors were analyzed using univariate and multivariate logistic regression models and survival analyses were performed using Kaplan-Meier. Menopause was considered to be associated with SF expression levels. The expression of CA125, CA153, and SF were associated with metastasis and FIGO stage. The expression of CA153 was associated with tumor grade. FIGO stage, menopause, expression levels of CA125 and CA153 are associated with poor prognosis in ovarian cancer patients. Through what we have found, nurses should pay attention to these prognostic factors in order to provide optimal nursing care and improve the quality of life of patients with ovarian cancer.
\end{abstract}

Keywords: nurse, ovarian cancer, clinicopathological feature, blood marker, prognosis

\section{INTRODUCTION}

The American Nursing Association defined nursing as "the diagnosis and treatment of human responses to existing or potential health problems". They stressed the importance of the "human behavior response", incorporating the physical, psychological, social, cultural, and spiritual aspects of behavioral responses. Nursing has always taken pride in adopting a holis-

$\Delta$ These authors contributed equally to this work and should be considered co-first authors.

*Correspondence to: Xujuan Xu, Department of Nursing, Nantong University Affiliated Hospital, Nantong, Jiangsu, 226001, China. Email: 353149945@qq.com; Xiuqun Xu, Department of Nursing, Nantong University Affiliated Hospital, Nantong, Jiangsu, 226001, China. E-mail: tyxiuqun@126.com.

The authors declare no conflicts of interest. tic or biopsychosocial approach that enables patients with individualized care. Nurses thus need education in many disciplines in order to practice effectively on a daily basis ${ }^{[1]}$. Ensuring that nurses are equipped with up-to-date scientific knowledge will improve their abilities to administer targeted therapies and help patients manage their personal health information for appropriate decision-making. For example, the dramatically increasing number of studies on genomics and oncology has highlighted urgent needs for nurses to have a good understanding of genetics ${ }^{[2,3]}$. Oncology nursing requires comprehensive knowledge, including genetics/genomics, to enhance the quality of care delivery ${ }^{[4]}$. Moreover, maintaining a patient-centered holistic approach also depends on the successful integration of disease- and intervention-related biomarkers throughout the nursing process.

The incidence of cancer is high worldwide ${ }^{[5]}$. Most 
nurses provide patient care in relation to treatment and health care in hospitals. In the case of cancer patients, nurses are involved in perioperative care, nursing radiation and chemotherapy related treatments, and terminal care. Some nurses work in the community and provide patient care outside of hospitals. Other nurses assist health care personnel, which may require a knowledge of the role of serum markers in cancer patients ${ }^{[3]}$, and an understanding of cancer screening and prognosis tracking in the community ${ }^{[6]}$.

Ovarian cancer is a common cancer that ranks the fifth among cancer-related causes of death in women ${ }^{[5]}$. Ovarian cancer presents a serious threat because its early symptoms are atypical, followed by rapid progress and early abdominal metastasis, resulting in $70 \%$ of patients presenting with advanced-stage disease ${ }^{[7]}$. Some cases detected early could undergo primary treatment, including surgery and chemotherapy, while advanced cases require targeted therapies ${ }^{[8-10]}$. Despite continuous developments in treatments of ovarian cancer, early diagnosis and treatment remain to be critical to improve patient prognosis. Gynecologic oncology clinicians and pathologists have carried out extensive research into tumor-associated markers, which will play an important role in the diagnosis of ovarian cancer together with imaging results ${ }^{[11,12]}$. Many studies have also focused on identifying the prognostic factors in ovarian cancer, with the aim of improving patient survival and quality of life. Factors like metastasis, cancer staging, and serum biomarkers have been shown to be associated with ovarian cancer prognosis ${ }^{[13-15]}$. In this study, we retrospectively collected clinical data from 103 ovarian cancer patients enrolled in our hospital and comprehensively evaluated the prognostic values of the clinicopathological features and frequentlyused blood markers, including carbohydrate antigen 125 (CA125), CA153, and serum ferritin (SF).

\section{MATERIALS AND METHODS}

\section{Patient information}

This retrospective study recruited 103 patients, who were diagnosed as ovarian cancer pathologically and had attended a single institution from May 2002 to May 2014. There were 72 cases of ovarian serous carcinoma (69.9\%), 14 cases of mucous cancer (13.5\%), 10 cases of endometrial carcinoma(9.7\%), and 7 cases of clear cell carcinoma $(6.9 \%)$. The median age at diagnosis was around 53 years old(range 32-79 years old). All the patients underwent standard surgery, including hysterectomy, bilateral salpingo-oophorectomy, pelvic and/or para-aortic lymphadenectomy and omentectomy. After surgery, patients were treated with platinum-based chemotherapy for at least six cycles. Patients' information was gathered from medical records, which included the results of blood tests, imaging studies, hospital progress notes, surgical records, pathology reports, diagnoses, general patient characteristics, clinical manifestations, menstrual history, obstetric history, and receipt of radiotherapy or chemotherapy. These patients were followed up by telephone to determine survival outcome. Follow-up started once a pathological diagnosis was made.

\section{Blood markers}

Blood samples were obtained two days before surgery, and none of the patients had received chemotherapy, radiotherapy, or immunotherapy before surgery. The collected blood samples were sent to the laboratory within 30 minutes for the detection of CA125, CA153, and SF. These serological markers were determined using chemiluminescence immunoassay. The positive value of CA125 was $>100 \mathrm{U} / \mathrm{mL}$. The positive value of CAl53 was $>100 \mathrm{U} / \mathrm{mL}$. The positive value of SF was $>400 \mathrm{ng} / \mathrm{mL}$.

\section{Follow-up}

The patients were followed up by a specialist nurse over the telephone monthly. Survival time was the period from the start of follow-up to the death of patients or the end of the study. Patients were staged according to the International Federation of Gynecology and Obstetrics (FIGO) staging system. The patients unable to take part in the follow-up were excluded from the study. The following retrospective data were analyzed in relation to follow-up outcomes, to identify factors affecting the prognosis of ovarian cancer: age, single-sided tumor, menopausal status, pathological type, clinical stage, pathological grade, serological markers (including CA125, CA153, SF), ascites, ascites tumor cells, lymph nodes, metastasis, and FIGO stage.

\section{Ethics and agreement}

Study protocol was approved by the Ethics Committee of the Affiliated Hospital of Nantong University, and all experiments were performed in accordance with approved guidelines of the Affiliated Hospital of Nantong University. All patients provided written informed consent.

\section{Statistical analysis}

The prognostic significance of the variables was analyzed by univariate analysis (log-rank test) and multivariate Cox regression model. Patients' survival curves were calculated using the Kaplan-Meier meth- 
od. All $P$ values were two-tailed, and a value of $P<$ 0.05 was considered to be statistically significant. All analyses were performed using SPSS 20.0 statistics software (SPSS Inc., Chicago, IL, USA) and STATA 12.0 (StataCorp, College Station, TX, USA).

\section{RESULTS}

All 103 patients were followed up until the death of patients or the final follow-up date of the study. The median follow-up duration was 49 months (range 2-83 months). We analyzed the associations between clinical factors and preoperative positive rates of blood markers, as shown in Table1. Menopause was significantly associated with raised positive rate of SF $(P=0.009)$; positive expression of CA125, CA153, and SF were more frequently found in patients with metastasis or FIGO stage III - IV ; and the positive rate of CA153 increased with tumor grade.

Univariate analysis of prognostic factors was performed using the Kaplan-Meier method and differences in survival rates were compared using logrank tests. Age $(P=0.014)$, menopause $(P=0.003)$, ascites tumor cells $(P=0.028)$, pathological stage $(P$ $=0.001)$, grade $(P=0.037)$, distant metastasis $(P=$ $0.001)$, CAl25 $(P=0.001)$, CAl53 $(P=0.012)$, and SF $(P=0.043)$ were significantly associated with a poorer prognosis (Table 2). According to the results of univariate analysis, factors like age, menopause, ascites tumor cells, pathological stage, grade, distant metastasis, CA125, CA153, and SF were associated with survival rate(Fig. 1).

Multivariate analysis was performed using a Cox proportional hazards regression model with multiple stepwise regression for each significant factor. Multiple regression analysis identified FIGO stage $(P=0.011)$, menopause $(P=0.005)$, CA125 $(P=0.043)$, and CAl53 $(P=0.039)$ as independent predictors of poorer prognosis in patients with ovarian cancer (Table 2).

\section{DISCUSSION}

\section{Staging and prognosis}

In this study, the clinical data of 103 patients with ovarian cancer were analyzed retrospectively to determine factors associated with prognosis. Many factors have been suggested to have prognostic values in ovarian cancer. For instance, Yoon et al. ${ }^{[16]}$ found that metastasis was associated with poor prognosis, while Zivanovic et al. ${ }^{[15]}$ suggested an association between pleural effusion and poor prognosis, and Kajiyama et al. ${ }^{[14]}$ identified ovarian cancer staging as an independent prognostic factor. In this study, FIGO stage, metastasis, ascites tumor cells, and tumor grade were related to prognosis in patients with ovarian cancer. These findings were consistent with previous results. Chinese women generally receive much fewer health examinations than those in developed countries. Moreover, the location of ovaries is in the depth of pelvis, causing the diagnosis in many ovarian can-

Table 1 Relationships between clinical features and tumor markers in patients with ovarian cancer

\begin{tabular}{|c|c|c|c|c|c|c|c|c|c|c|c|c|c|}
\hline \multirow{2}{*}{ Clinical features } & & \multicolumn{4}{|c|}{ CA125 } & \multicolumn{4}{|c|}{ CA153 } & \multicolumn{4}{|c|}{ SF } \\
\hline & & $n$ & Positive/n $(\%)$ & $\chi^{2}$ & $P$ & $n$ & Positive/n $(\%)$ & $\chi^{2}$ & $P$ & $n$ & Positive/n $(\%)$ & $\chi^{2}$ & $P$ \\
\hline \multirow[t]{3}{*}{ Age (years) } & $<60$ & 54 & $40(74.07)$ & 0.758 & 0.384 & 57 & $28(49.12)$ & 0.196 & 0.658 & 53 & $11(20.75)$ & 3.769 & 0.005 \\
\hline & $\geqslant 60$ & 41 & 27 (65.85) & & & 41 & $22(53.66)$ & & & 41 & $16(39.02)$ & & \\
\hline & Unknown & 8 & & & & 5 & & & & 9 & & & \\
\hline \multirow[t]{3}{*}{ Menopause } & No & 31 & $21(67.74)$ & 0.083 & 0.773 & 33 & $16(48.48)$ & 0.292 & 0.589 & 32 & $4(12.50)$ & 6.786 & 0.009 \\
\hline & Yes & 58 & $41(70.69)$ & & & 57 & $31(54.39)$ & & & 54 & $21(38.89)$ & & \\
\hline & Unknown & 14 & & & & 13 & & & & 17 & & & \\
\hline \multirow[t]{3}{*}{ Grade } & 0 & 26 & $17(65.38)$ & 0.546 & 0.460 & 27 & $8(29.63)$ & 5.942 & 0.015 & 25 & $4(16.00)$ & 2.950 & 0.086 \\
\hline & 1 & 67 & $49(86.05)$ & & & 68 & $39(57.35)$ & & & 67 & $23(34.33)$ & & \\
\hline & Unknown & 10 & & & & 8 & & & & 11 & & & \\
\hline \multirow[t]{3}{*}{$\begin{array}{l}\text { Ascites } \\
\text { tumor cells }\end{array}$} & No & 46 & $30(65.22)$ & 0.533 & 0.465 & 46 & $23(50.00)$ & 1.435 & 0.231 & 44 & $10(22.73)$ & 2.365 & 0.124 \\
\hline & Yes & 23 & $17(73.91)$ & & & 23 & $15(65.22)$ & & & 22 & $9(28.79)$ & & \\
\hline & Unknown & 34 & & & & 34 & & & & 37 & & & \\
\hline \multirow[t]{3}{*}{ Lymph nodes } & No & 75 & $51(68.00)$ & 0.624 & 0.430 & 77 & 38 (49.35) & 0.090 & 0.764 & 72 & $20(27.78)$ & 0.241 & 0.623 \\
\hline & Yes & 14 & $11(78.57)$ & & & 13 & 7 (53.85) & & & 14 & $3(21.43)$ & & \\
\hline & Unknown & 14 & & & & 13 & & & & 17 & & & \\
\hline \multirow[t]{3}{*}{ Metastasis } & No & 49 & 29 (59.18) & 6.263 & 0.012 & 52 & 20 (38.46) & 6.992 & 0.008 & 50 & $8(16.00)$ & 8.446 & 0.004 \\
\hline & Yes & 46 & 38 (82.61) & & & 46 & $30(65.22)$ & & & 44 & $19(43.18)$ & & \\
\hline & Unknown & 8 & & & & 5 & & & & 9 & & & \\
\hline \multirow[t]{3}{*}{ FIGO } & I - II & 52 & $30(57.69)$ & 9.103 & 0.003 & 55 & $22(40.00)$ & 6.092 & 0.014 & 53 & $9(16.98)$ & 8.184 & 0.004 \\
\hline & III - IV & 43 & $37(86.05)$ & & & 43 & $28(65.12)$ & & & 41 & $18(43.90)$ & & \\
\hline & Unknown & 8 & & & & 5 & & & & 9 & & & \\
\hline
\end{tabular}


Table 2 Cox proportional hazards regression model

\begin{tabular}{lccccccc}
\hline \multirow{2}{*}{ Factors } & \multicolumn{3}{c}{ Univariate analysis } & & \multicolumn{3}{c}{ Multivariate analysis } \\
\cline { 2 - 3 } & HR & $P$ & $95 \%$ CI & & HR & $P^{*}$ & \\
Age & 2.027 & 0.014 & $1.152-3.567$ & & & \\
Menopause & 2.886 & 0.003 & $1.429-5.827$ & & 3.466 & 0.005 & $1.456-8.252$ \\
Type & 0.909 & 0.474 & $0.699-1.181$ & & 1.154 & 0.391 & $0.832-1.599$ \\
Grade & 2.239 & 0.037 & $1.049-4.782$ & & 1.230 & 0.678 & $0.463-3.272$ \\
CA125 & 4.236 & 0.001 & $1.794-10.005$ & & 2.578 & 0.043 & $1.030-6.454$ \\
CA153 & 2.151 & 0.012 & $1.180-3.921$ & & 2.267 & 0.039 & $1.041-4.934$ \\
SF & 1.854 & 0.043 & $1.019-3.373$ & & 0.966 & 0.930 & $0.451-2.071$ \\
Ascites & 1.689 & 0.089 & $0.924-3.087$ & & N/A & & \\
Ascites tumor cells & 2.029 & 0.028 & $1.081-3.808$ & & N/A & & \\
Lymph nodes & 1.903 & 0.073 & $0.942-3.846$ & & N/A & & \\
Metastasis & 5.571 & 0.001 & $7.944-10.543$ & & N/A & & \\
FIGO & 4.242 & 0.001 & $2.330-7.724$ & & 2.943 & 0.011 & $1.283-6.752$ \\
\hline
\end{tabular}

"adjusted for age. HR, hazard ratio; CI, confidence interval. N/A, not applicable. Ascites, ascites tumor cells, lymph nodes, and metastasis did not enter multivariate analysis as independent factors, since these factors were integrated into FIGO.


Fig. 1 Kaplan-Meier analyses (log-rank test) of survival. (a) Overall survival (OS) was significantly lower in patients aged $\geqslant 60$ years compared with those aged $<60$ years $(P=0.012 .1$ means age $\geqslant 60$ years; 0 means age $<60$ years. (b) OS was significantly lower in postmenopausal compared with premenopausal patients $(P=0.002)$. 1 means postmenopausal; 0 means premenopausal.(c) OS was significantly lower in patients with ascites tumor cells compared with those with no ascites tumor cells $(P=0.023)$. 1 means ascites tumor cells; 0 means no tumor cells in ascites. $(\mathrm{d})$ OS was significantly lower in patients with CA125 expression compared with those without CA125 expression $(P<0.001)$. 1 means CA125 is higher than $100 \mathrm{U} / \mathrm{mL} ; 0$ means that CA125 is below $100 \mathrm{U} / \mathrm{mL}$. (e) OS was significantly lower in patients with CA153 expression compared with those without CA153 expression $(P=0.010)$. 1 means CA153 is higher than $100 \mathrm{U} / \mathrm{mL} ; 0$ means that CA153 is below $100 \mathrm{U} / \mathrm{mL}$. (f) OS was significantly lower in patients with SF expression compared with those without SF expression $(P=0.039)$. 1 means SF above $400 \mathrm{ng} / \mathrm{mL} ; 0$ means SF below $400 \mathrm{ng} / \mathrm{mL}$. $(\mathrm{g})$ OS was significantly lower in patients with metastasis compared with those without metastasis $(P<0.001)$. 1 means lymph node metastasis; 0 means without lymph node metastasis. (h) OS was significantly lower in patients with low-grade compared with high-grade tumors $(P=0.031)$. 1 means low-grade tumor; 0 means high-grade tumor. (i) OS was significantly lower in patients with FIGO III - IV compared with FIGO I - II $(P<0.001)$. 1 means FIGO III - IV ; 0 meaning FIGO I - II . 
cer patients' cancer to be missed in its early stages. Consequently, most of cases are only detected at an advanced stage, by which time treatment options are limited. Compared with advanced tumors, early ovarian cancers can be treated surgically with relatively smaller residual tumors postoperatively, high sensitive to chemotherapy with a low incidence of resistance, and lower recurrence rate. Thus the more advanced the stage, the poorer the prognosis. We recommend that working women should be provided with a health checkup every year by employers. Women who have no work units should be provided with a health physical examination once a year by the government. The contents of the physical examination should include: gynecological examination, gynecological B superposition, and blood markers related to gynecological tumors. It is expected that ovarian cancer can be found early and patients can get early treatment, thus having a longer survival period.

\section{Blood markers and prognosis}

Immune serum tumor markers can be used to detect and monitor tumors ${ }^{[17,18]}$. CA153 has been used to diagnose breast cancer ${ }^{[19]}$, as well as lung cancer and pleural effusion ${ }^{[20]}$. In this study, we demonstrated that CA153 expression level was also an independent prognostic factor in ovarian cancer. It is possible that patients diagnosed with advanced ovarian cancer may already have lung metastasis, accounting for the increased levels of CA153. CA125 has been shown to play an important role in diagnosis of ovarian cancer and ovarian cancer relapse ${ }^{[21,22]}$. In the present study, CA125 expression was correlated with ovarian cancer prognosis which is consistent with the findings of other studies ${ }^{[23]}$.

\section{Age and prognosis}

The relationship between age and ovarian cancer prognosis is unclear. Some studies ${ }^{[24]}$ found no significant relationship between age and prognosis in ovarian cancer patients, while others ${ }^{[7,25,26]}$ have suggested that older patients have a poorer prognosis than younger patients. The relationship between age and prognosis may reflect greater potential for postoperative recovery and sensitivity of chemotherapy in younger patients. In this study, menopause was identified as an independent prognostic factor. This correlation may be related to age because women in China undergo menopause around 45-55 years old, and most ovarian cancer patients were detected during this age. Further studies with large sample sizes are needed to clarify the association between age, menopause and prognosis of ovarian cancer in China. If possible, se- rum levels of relevant biomarkers should also be dynamically monitored in ovarian cancer patients during follow-up.

\section{Nursing services and prognosis}

It is imperative for oncology nurses to be aware of the progress in the prognosis of ovarian cancer. Clinical nurse specialists are professional caregivers who provide all aspects of care and encourage a close relationship between the cancer patient and his/her family. It has been recognized that the appointment of senior clinical nurse specialists to work together with doctors can provide more specialized patient care from which patients will be benefited much. In China, the increasing international communication facilitated by the Internet has resulted in rapid developments in the field of nursing over the past decades. The important roles of nurses in caring patients with cancer have been more and more emphasized. Nurses care for patients presenting with all stages of cancer, and are involved in the diagnosis, treatment and prognosis ${ }^{[27-30]}$. They also provide the patients with information relating to their disease, as well as their general health and prognosis ${ }^{[31,32]}$. Nurses thus use their clinical experience and knowledge of prognosis to provide health guidance to their patients. In China, most ovarian cancer patients are not detected until they are in advanced stages, which is partially due to the failure of screening for cancer nationwide and women's unawareness of the importance of regular health examination. Thus far, detection, diagnosis, and treatment early are the only realistic means to improve ovarian cancer survival rate. A well-educated nurse with knowledge of prognostic factors in patients with ovarian cancer can not only help patients manage their conditions and improve the quality of life better but also make patients and relatives appreciate the significance of gynecological examinations and regular health checks. Literature mentions that hospital volume and nursing quality are associated with survival rates of ovarian cancer patients and patients having high-quality care are likely to have higher survival rates ${ }^{[33]}$. We believe that nurses should provide nursing care for patients with ovarian cancer, such as arranging patients to be admitted to hospital, ordering hospitalization, providing high-quality nursing care from the patients' first diagnosis. Nursing means an integrated and continuous process, rather than being split into hospital nursing, surgical nursing, discharge guidance hospital care. For hospital nursing is not the end of care for ovarian cancer patients, nurses should provide continuing care services. The nurses working in hospitals can collaborate with those in the community and provide a 
co-managed service for ovarian cancer patients after discharge. They can monitor the course of illness, remind survivors to go to the hospital for review in time and provide necessary support and help. Nurses can organize survivors' participation in various activities, such as communicating with cancer patients, doing aerobic exercise, etc, which will encourage them to build up their confidence and courage in fighting cancer. Nurses can go to the survivors' home, assess their health and family social support systems dynamically, and provide personalized nursing care.

In summary, this study provided evidence that FIGO stage, CA125 and CA153 expression levels, and menopause might contribute to poor prognosis in ovarian cancer patients. The results of this study show ways that nurses could be supported more in their nursing role thus improving the prognosis of patients with ovarian cancer. High-quality nursing care is important for patients when treated in the hospital. Nurses need to master the knowledge of the factors affecting the prognosis of patients with ovarian cancer, and guide people, encourage all adult women to participate in physical examination, especially the special inspection (gynaecological examination, B ultrasonic inspection, tumor markers, etc.), so that we can find early cases, and the patient is able to receive early treatment. When the patient's treatment is over, the nurse should provide continuous nursing care through the cooperative nursing mode and administer various services to the survivors in order to extend the life period and improve the life quality.

\section{Acknowledgments and funding}

This study was supported by the Social Development and Applied Research Projects of Nantong (S7944), and the Technological Innovation and Demonstration of Social Undertakings Projects of Nantong (MS22015093).

\section{References}

[1] Calzone KA, Cashion A, Feetham S, et al. Nurses transforming health care using genetics and genomics. Nurs Outlook, 2010, 58(1):26-35.

[2] Daack-Hirsch S, Dieter C, Quinn Griffin MT. Integrating genomics into undergraduate nursing education. $J$ Nurs Scholarsh, 2011, 43(3):223-30.

[3] Eggert J. The biology of cancer: what do oncology nurses really need to know. Semin Oncol Nurs, 2011, 27(1):3-12.

[4] Charalambous A, Adamakidou T, Cloconi C, et al. The quality of oncology nursing care: A cross sectional survey in three countries in Europe. Eur J Oncol Nurs,2017,27(1):45-52.

[5] Jones MR, Kamara D, Karlan BY, et al. Genetic epide- miology of ovarian cancer and prospects for polygenic risk prediction. Gynecol Oncol,2017,147(3):705-13.

[6] Tu SP, Young VM, Coombs LJ, et al. Practice adaptive reserve and colorectal cancer screening best practices at community health center clinics in 7 states. Cancer,2015,121(8):1241-8.

[7] Hou MM, Chen Y, Wu YK, et al. Pathological characteristics and prognosis of 664 patients with epithelial ovarian cancer: a retrospective analysis. Sichuan Da Xue Xue Bao(in Chinese),2014,45(5):859-75.

[8] Banerjee S, Kaye SB. New strategies in the treatment of ovarian cancer: current clinical perspectives and future potential. Clin Cancer Res,2013,19(5): 961-8.

[9] Cheng L, Wu S, Zhang K, et al. A comprehensive overview of exosomes in ovarian cancer: emerging biomarkers and therapeutic strategies. J Ovarian Res,2017, 10(1):73.

[10] Schmid BC, Oehler MK. New perspectives in ovarian cancer treatment. Maturitas,2014,77(2):128-36.

[11] Grant P, Sakellis C, Jacene HA. Gynecologic oncologic imaging with PET/CT. Semin Nucl Med, 2014,44(6): 461-78.

[12] Felder M, Kapur A, Gonzalez-Bosquet J, et al. MUC16 (CA125): tumor biomarker to cancer therapy, a work in progress. Mol Cancer,2014,13(1):129.

[13] Drescher CW, Shah C, Thorpe J, et al. Longitudinal screening algorithm that incorporates change over time in CA125 levels identifies ovarian cancer earlier than a single-threshold rule. J Clin Oncol,2013,3(31): 38792.

[14] Kajiyama H, Shibata K, Mizuno M, et al. Long-term clinical outcome of patients with recurrent epithelial ovarian carcinoma: is it the same for each histological type? Int J Gynecol Cancer,2012, 22(3):394-9.

[15] Zivanovic O, Barakat RR, Sabbatini PJ, et al. Prognostic factors for patients with stage IV epithelial ovarian cancer receiving intraperitoneal chemotherapy after secondlook assessment: results of long-term follow-up. Cancer,2008, 112(12):2690-7.

[16] Yoon A, Park JY, Lee YY, et al. Prognostic factors and outcomes in endometrial stromal sarcoma with The 2009 FIGO staging system: a multicenter review of 114 cases. Gynecol Oncol,2014,132(1):70-5.

[17] Lellé RJ, Henkel E, Leinemann D, et al. Measurement of CEA, TPA, neopterin, CA125, CA153 and CA199 in sera of pregnant women, umbilical cord blood and amniotic fluid. Gynecol Obstet Invest,1989,27(3): 137-42.

[18] Wang XF, Wu YH, Wang MS, et al.CEA, AFP, CA125, CA153 and CA199 in malignant pleural effusions predict the cause. Asian Pac J Cancer Prev,2014, 15(1):363-8.

[19] Zheng H, Luo RC. Diagnostic value of combined detection of TPS, CA153 and CEA in breast cancer. Di Yi Jun Yi Da Xue Xue Bao (in Chinese), 25(10): 1293-8.

[20] Wu G, Ba J, Wang E, et al. Diagnostic value of determination of CEA, CA125, CA153 and CA199 assay in pleural fluid for lung cancer. Zhongguo Fei Ai Za Zhi (in Chinese),2004,7(1):35-7. 
[21] Kristjansdottir B, Levan K, Partheen K, et al. Diagnostic performance of the biomarkers HE4 and CA125 in type I and type II epithelial ovarian cancer. Gynecol Oncol,2013, 131(1):52-8.

[22] Song MJ, Lee SH, Choi MR, et al. Diagnostic value of CA125 as a predictor of recurrence in advanced ovarian cancer. Eur J Gynaecol Oncol,2013,34(2):148-51.

[23] Anastasi E, Porpora MG, Pecorella I, et al. May increased CA125 in borderline ovarian tumor be indicative of a poor prognosis? A case report. Tumour Biol,35(7):6969-71.

[24] Tang L, Zheng M, Xiong Y, et al. Clinical characteristics and prognosis of epithelial ovarian cancer in young women. Chinese Journal of Cancer (in Chinese),2008,27(9):951-5.

[25] Jorgensen TL, Teiblum S, Paludan M, et al. Significance of age and comorbidity on treatment modality, treatment adherence, and prognosis in elderly ovarian cancer patients. Gynecol Oncol,2012,127(2): 367-74.

[26] Maas HA, Kruitwagen RF, Lemmens VE, et al. The influence of age and co-morbidity on treatment and prognosis of ovarian cancer: a population-based study. Gynecol Oncol,2005,97(1):104-9.

[27] Leary A, Baxter J. Impact of lung cancer clinical nurse specialists on emergency admissions. Br J Nurs,2014,
23(17):935-8.

[28] Babine RL, Honess C, Wierman HR, et al. The role of clinical nurse specialists in the implementation and sustainability of a practice change. J Nurs Manag,2016,24(11):39-49.

[29] Klinkner G, Murray M. Clinical nurse specialists lead teams to impact glycemic control after cardiac surgery. Clin Nurse Spec,2014, 28(4):240-6.

[30] Clark JE, Aitken S, Watson N, et al. Training oncology and palliative care clinical nurse specialists in psychological skills: Evaluation of a pilot study. Palliat Support Care,2015,13(3):537-42.

[31] Ream E, Wilson-Barnett J, Faithfull S, et al. Working patterns and perceived contribution of prostate cancer clinical nurse specialists: a mixed method investigation. Int J Nurs Stud,2009, 46(10):1345-54.

[32] Parkinson N, Pratt H. Clinical nurse specialists and the psychosexual needs of patients with gynaecological cancer. J Br Menopause Soc,2005,11(1):33-5.

[33] Wright JD, Chen L, Hou JY, et al. Association of hospital volume and quality of care with survival for ovarian cancer. Obstet Gynecol, 2017,130(3):545-53.

(Received 22 November 2017, Revised 07 December 2017, Accepted 11 December 2017) 